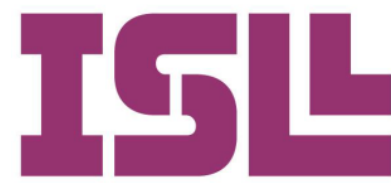

Número 4.

Julio de 2015

\title{
La formación de los futuros maestros a través de la literatura: los textos literarios desde una perspectiva didáctico-práctica
}

\author{
María Victoria Guadamillas Gómez \\ Universidad de Castilla-La Mancha
}

Pág. 93 a la 106

\section{Abstract:}

The aim of this contribution is to

Thirdly, this article shows the context

\section{Keywords}

Children's Literature, Curriculum Design, Didactics, Literature Teaching, Teacher training reflect on the training on literary issues provided to future teachers, particularly, on Children's Literature at Higher Education. To this end, a qualitative study has been conducted by means of the implementation of a didactic experience at the Faculty of Education in Toledo with the students of the Degree in Infant Education at University of CastillaLa Mancha. Through this didactic experience, students were assumed to gain knowledge of the main contributions to Children's Literature in the context of the elective course Children's Literature in English and its Didactics. Firstly, some advantages of the inclusion of Children's Literature and its possibilities at early educational levels are revised through some of the most relevant authors on the field. Secondly, this paper pays attention to the necessity of including Children's Literature in Infant Education Studies, giving its importance in early educational levels. of the study, particularly, the approach followed in the course as well as its main objectives, contents, learning outcomes and assessment criteria implemented. Once the main aspects of the study have been described, including its design, methodology and participants, this paper focuses on the analysis of the results obtained in the qualitative study conducted. Finally, this article considers the participants' opinion and their satisfaction rate obtained from the results of the study at the end of the didactic intervention carried out in the first semester of the Academic Year 2015-2016. 


\section{Introducción}

La literatura infantil es un recurso de gran importancia en las primeras etapas educativas, existiendo diferentes conexiones entre esta y el desarrollo lingüístico y emocional de los niños que nos dan muestra de su magnitud. La literatura se relaciona tanto con el proceso de adquisición y desarrollo de la lengua materna, como con el proceso inicial de aprendizaje de la segunda lengua y su uso y disfrute en el aula queda reflejado en los reales decretos y decretos autonómicos para el segundo ciclo de Educación Infantil y para Educación Primaria.

Numerosos estudios se fijan en el tratamiento de la literatura infantil en las etapas iniciales (Selfa i Sastre et al., 2015). Por su parte, Villacañas de Castro (2013) se refiere a la literatura y el aprendizaje de la L2 en la educación superior y realiza un estudio del tratamiento de los textos infantiles en lengua inglesa, incidiendo en los beneficios lingüísticos de estos. La reciente contribución de Caballud y Carramiñana (2015) va más allá, refiriéndose al proceso de formación permanente a través de grupos de trabajo en los que se estudie el potencial de los recursos literarios con el fin de elaborar planes de lectura, escritura y comprensión en los que también sean incluidos las familias.

Otras aportaciones, como la de Cameron (2001), se fijan en características más concretas presentes en los textos infantiles. La autora destaca que la literatura infantil proporciona numerosos recursos de gran importancia en el proceso de aprendizaje de la segunda lengua, destacando, entre otros, los paralelismos o la riqueza del lenguaje empleado. Para dar cuenta del segundo aspecto, la autora realiza un estudio de la historia On the Way Home (2007) de Jill Murphy y pone atención al uso de los verbos de movimiento en las acciones de los personajes: "zooming, lumbering, slithering, soaring, creeping, gliding...etc." (Cameron, 2001, p.163). Otros aspectos que la autora recalca son la presencia de metáforas o la aliteración, consistente esta última en la repetición de sonidos iniciales a través de las palabras y de frecuente uso en las nursery rhymes (rimas infantiles).

Gracias a estas y otras cuestiones, la formación literaria representa un pilar fundamental en la preparación de los futuros maestros y, así, los planes de estudios del Grado de Maestro en Educación Infantil y Primaria vigentes en muchas universidades españolas contemplan asignaturas relacionadas con la literatura y su didáctica. En el caso de la Universidad de Castilla-La Mancha, escenario en el que se desarrolla nuestro estudio, el plan de Graduado en Maestro de Educación Infantil contiene 6 créditos obligatorios para la materia de Literatura Infantil y Animación a la Lectura y 6 créditos optativos para los maestros que opten por el itinerario de Lengua Extranjera: Inglés, en la materia Literatura Infantil en Lengua Inglesa y su Didáctica.

ISL, vol. 4, 2015, págs. 93-106 ISNN: $2340-8685$
Guadamillas Gómez, M. V. (2015): La formación de los futuros maestros a través de la literatura: los textos literarios desde una perspectiva didáctico-práctica, Investigaciones Sobre Lectura, 4, 93-106. 
En el caso de la formación inicial, la literatura está presente en los currículos de Educación Infantil vigentes en España. En concreto dentro del decreto 67/2007 de 29 de mayo, vigente en la comunidad autónoma de Castilla-La Mancha y encuadrado en el área: Los lenguajes: comunicación y representación, donde se incluye un bloque que hace referencia al lenguaje verbal y dentro del cual se encuadran contenidos relacionados con la escritura y la lectura a través de cuentos, libros ilustrados, rimas o canciones. Sin embargo, la importancia de incluir la literatura infantil en el aula de Educación Superior no solo viene dada por los currículos que los futuros maestros deben desarrollar en la escuela infantil, esta es también relevante desde un punto de vista lingüístico, ya que les permite desarrollar la segunda lengua dentro de un contexto creativo donde la comunicación y otros aspectos culturales emergen de manera sencilla. Desde este punto de vista, la literatura infantil facilita la exposición a material auténtico a través del cual se pueden desarrollar las destrezas lingüísticas: listening, reading, writing o speaking.

En este sentido, algunos estudios como de los Khatib et al., (2011) manifiestan que través de la literatura pueden ser trabajados: "different aspects of language authentically within itself including not only syntax and lexis but also pragmatic and cultural points not easily found in non-literary texts" (Khatib et al., 2011, p.215). La motivación en el aula es otro de los aspectos que la mayoría de los estudios señalan, siendo algunos de los más relevantes los de Maley (2001) o Van (2009). Maley se refiere a algunas de los beneficios que presenta la literatura en la instrucción en segunda lengua, destacando: "su autenticidad, universalidad o el desarrollo del vocabulario y otros aspectos interculturales" (Maley, 2001, p.182). Por su parte, Van (2009) menciona el papel de la literatura para trabajar los aspectos interculturales y reflexionar sobre estos con los estudiantes.

Es por todo ello que los maestros han de conocer, leer y comprender los textos más relevantes de este género, destacando las rimas, los libros ilustrados o los cuentos. Sin embargo, no es de menor importancia el hecho de que los estudiantes necesitan aprender a usar los textos en el aula de Educación Infantil. En este sentido, tratamos de dar a la formación de los futuros maestros un "enfoque clínico" que como Andreucci (2012) define: "se centra en el desarrollo vocacional- profesional del docente mediante una relación democrática y colaborativa realizada in situ, garantizando la significación práctica que para el profesor tienen estos procesos" (Andreucci, 2012, p.261).

En este contexto y siguiendo este enfoque clínico, queremos averiguar si los estudiantes están satisfechos con la orientación didáctico-práctica de la materia y si perciben sus principales aportaciones, contando para ello con los siguientes objetivos específicos: (1) conocer la importancia de la asignatura Literatura Infantil en Lengua Inglesa y su Didáctica dentro del plan de estudios del Grado de Maestro en Educación Infantil, (2) saber si la materia incrementa el gusto de los estudiantes por las diferentes 
manifestaciones literarias escritas en lengua inglesa, (3) corroborar cuáles son las principales competencias que los estudiantes desarrollan a lo largo de las actividades planteadas en la materia, (4) conocer las conexiones entre lo tratado en esta materia y el desarrollo de su profesión como maestros y (5) percibir si la asignatura ha contribuido a incrementar el interés por el uso y lectura de textos literarios fuera de lo estrictamente necesario y aplicado en la materia. De forma más general, tratamos de averiguar también a través de los estudiantes los aspectos más positivos y negativos durante el periodo formativo.

\section{Método: diseño y desarrollo de la investigación}

\subsection{Descripción del estudio y sus participantes}

El estudio ha sido llevado a cabo en el aula de la materia Literatura Infantil en Lengua Inglesa y su Didáctica durante el primer cuatrimestre del curso 2013-2014. Los participantes son 20 estudiantes del Grado de Maestro en Educación Infantil y de cuarto curso, tres de los estudiantes son de nacionalidad polaca y cursan la materia dentro del programa Erasmus + que permite la movilidad de estudiantes dentro de la Unión Europea. Con el objetivo de conocer cuáles son los beneficios que los maestros en formación adquieren para su futuro profesionales a través de la asignatura objeto de estudio y cómo estos mismos perciben el programa formativo y el diseño de actividades desarrollado en la materia, planteamos cuestionarios en los que, anónimamente, los estudiantes valoren cuestiones generales y específicas relacionadas con el desarrollo de la materia, su desarrollo y su grado de acuerdo con lo que el profesor considera se ha adquirido.

Se trata de un estudio cualitativo y descriptivo que pretende mostrar el nivel de satisfacción del alumnado participante en la orientación didáctica de la asignatura con base literaria. El estudio cualitativo resulta uno de los más efectivos en investigación educativa y permite además: "un contacto directo entre el investigador y el objeto de la investigación" (Hernández Castilla y Opazo Carvajal, 2010, p.5). El instrumento para nuestro estudio es un cuestionario que cuenta con cinco preguntas de respuesta cerrada para conocer la satisfacción general de los estudiantes con la materia, las principales aportaciones de este curso a la preparación de los futuros maestros y los nuevos conocimientos adquiridos a través de la materia. Además, se añade una última pregunta de carácter abierto y en la que pedimos a los estudiantes que señalasen los aspectos positivos y negativos del curso. Asimismo, los estudiantes realizaron tres cuestionarios más siguiendo el mismo formato y que pretendían conocer, en particular, la valoración de las actividades con bases didáctica realizadas en torno a los diferentes géneros, estos serán analizados en literatura sucesiva y no son objeto de análisis en este trabajo.

ISL, vol. 4, 2015, págs. 93-106 ISNN: 2340-8685
Guadamillas Gómez, M. V. (2015): La formación de los futuros maestros a través de la literatura: los textos literarios desde una perspectiva didáctico-práctica, Investigaciones Sobre Lectura, 4, 93-106. 


\subsection{Contexto: descripción del enfoque didáctico de la asignatura objeto de estudio}

La asignatura en la que llevamos a cabo este estudio cuenta con un total de 6 créditos y es de carácter cuatrimestral. De acuerdo con la guía docente seguida en el curso 2014-2015 y con vocación de continuidad en el curso 2015-2016, los alumnos cuentan con 100 horas presenciales, más 50 de trabajo autónomo; las primeras se agrupan en dos bloques de dos horas semanales. En cuanto a los resultados del aprendizaje esperados pretendemos que los alumnos: (1) comprendan las ideas principales e información específica en mensajes escritos, principalmente, literarios o narrativos de una cierta extensión, (2) sepan comunicarse con hablantes nativos y no nativos en lengua inglesa con un cierto grado de fluidez, sobre diversos temas didácticos o literarios que aparezcan en el aula, siendo capaces de seguir la línea argumental de estos mensajes (3) sepan programar sesiones y unidades didácticas basadas en materiales literarios tales como cuentos, chants o canciones, teatro o libros ilustrados. Por último, valoramos también que los estudiantes (4) sean capaces de presentar estas actividades en lengua inglesa en el aula.

Para contribuir al desarrollo de los resultados esperados, los contenidos se agrupan en seis grandes bloques. El primero de ellos tiene carácter general y pretende concienciar a los estudiantes de la importancia de la literatura infantil en las primeras etapas educativas, principalmente, en educación infantil. En el segundo bloque nos referimos a los orígenes de la literatura infantil realizando un breve recorrido histórico a través de las principales obras pertenecientes a este género. Los bloques tercero, cuarto, quinto y sexto tienen un enfoque similar y se centran en las rimas infantiles, los libros ilustrados, la narrativa y otros géneros, respectivamente. Se presentan algunas de las obras más representativas pertenecientes a estos en lengua inglesa y a partir de la lectura de los textos, los estudiantes desarrollan intervenciones didácticas breves dirigidas al segundo ciclo de Educación Infantil y basadas en ellos, para culminar con el desarrollo de una unidad didáctica basada en una obra narrativa infantil inglesa.

La materia tiene carácter presencial y plantea diferentes actividades que los alumnos deben realizar. Para la poesía, los libros ilustrados, el cuento y el teatro, los estudiantes deben preparar una intervención didáctica breve en la que muestren alguno de los materiales literarios tratados durante las sesiones. Han de leer cuatro lecturas obligatorias y realizar un proyecto final que verse sobre una de ellas y en la que presenten al menos cuatro sesiones de trabajo e incluyan objetivos, contenidos, desarrollo y evaluación, así mismo, y fomentando la creatividad y la expresión oral y escrita en lengua inglesa han de presentar oralmente y dramatizado un nuevo final para la historia objeto de estudio, este trabajo deberá entregarse también por escrito en lengua inglesa. Por último, la asignatura Literatura Infantil en Lengua Inglesa y su Didáctica cuenta con una prueba final de carácter teórico-práctico sobre los contenidos tratados en la materia. 
El sistema de evaluación es presencial y se asignan los siguientes porcentajes a cada una de las actividades:

\begin{tabular}{|l|c|}
\hline \multicolumn{1}{|c|}{ ACTIVIDAD } & PORCENTAJE \\
\hline Proyecto final & $40 \%$ \\
\hline Test final & $30 \%$ \\
\hline Intervenciones cortas: poesía, libro ilustrado, cuento, teatro & $5 \%$ cada una. Total $20 \%$ \\
\hline Dramatización oral y entrega escrita de un nuevo final & $10 \%$ \\
\hline Total & $100 \%$ \\
\hline
\end{tabular}

Tabla 1. Sistema de evaluación de la materia Literatura Infantil en Lengua Inglesa y su Didáctica (20142015)

Así pues, y como se ha expuesto anteriormente, pretendemos combinar los aspectos teóricos y los que tienen que ver con un enfoque didáctico tanto a través de los contenidos como al establecer el proceso de evaluación en la materia, destacando el hecho de que las actividades prácticas realizadas por el alumnado son objeto de evaluación. Esto nos permite que los estudiantes incrementen su motivación para la lectura de textos literarios que, en ocasiones, pueden resultar complejos y de tediosa lectura para los futuros maestros.

\subsection{Análisis de resultados}

En este apartado nos referimos a los resultados obtenidos en el primer cuestionario de carácter general del que participaron un total de 20 alumnos, los cuales respondieron de manera anónima a las preguntas planteadas. A través de la primera pregunta pretendemos responder al primer objetivo de nuestra investigación, los estudiantes consideraban la importancia de la asignatura Literatura Infantil en Lengua Inglesa y su Didáctica dentro de su formación global como maestros.

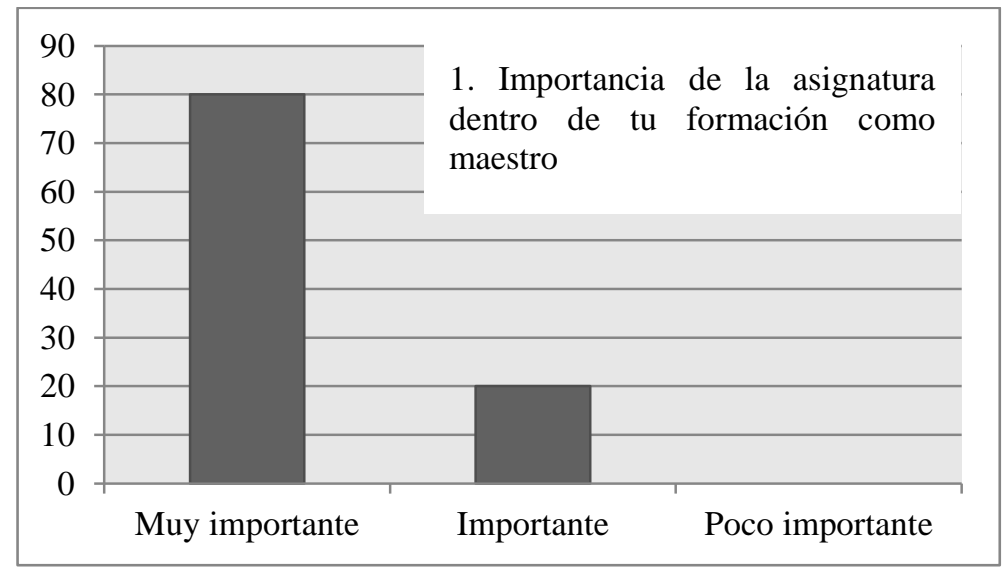

Figura 1. Respuesta 1 del cuestionario: importancia de la asignatura de Literatura Infantil en LI y su Didáctica dentro de tu formación

Un $80 \%$ de los estudiantes que respondieron al cuestionario considera muy importante la materia, mientras que un $20 \%$ cree que esta formación es importante

ISL, vol. 4, 2015, págs. 93-106 ISNN: 2340-8685
Guadamillas Gómez, M. V. (2015): La formación de los futuros maestros a través de la literatura: los textos literarios desde una perspectiva didáctico-práctica, Investigaciones Sobre Lectura, 4, 93-106. 
dentro de su curriculum formativo. Ninguno de los alumnos consideró poco importante la asignatura objeto de estudio. Preguntados en la segunda pregunta del cuestionario sobre si la asignatura, de manera general, ha contribuido a aumentar su interés por la literatura escrita en lengua inglesa, el $100 \%$ de los encuestados respondieron afirmativamente como se muestra en la siguiente figura:

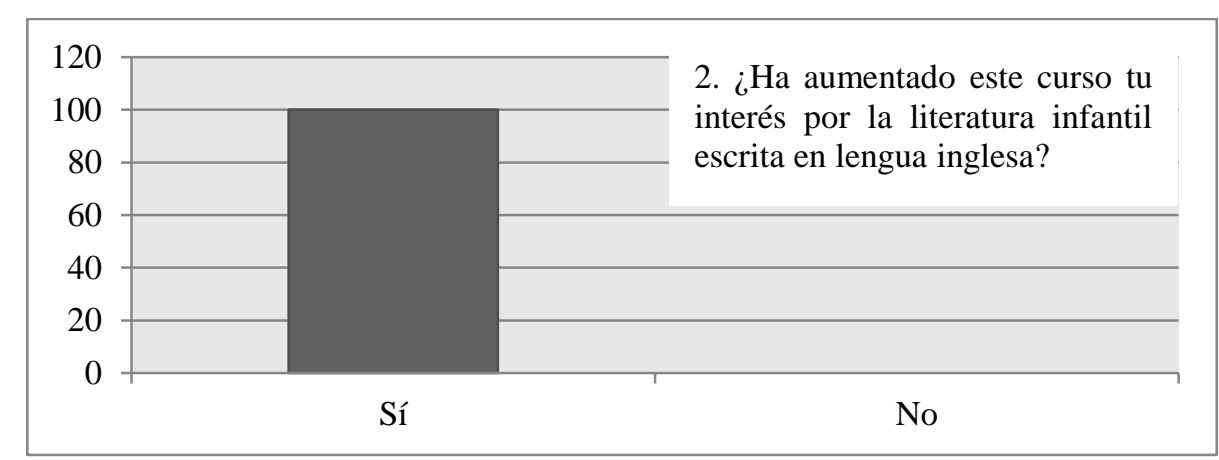

Figura 2. Pregunta 2 del cuestionario: ¿Ha aumentado este curso tu interés por la literatura escrita en lengua inglesa?

En la tercera pregunta del cuestionario, los encuestados tenían que elegir una de las cuatro opciones disponibles que respondiesen a cuáles habían sido los principales contenidos o habilidades adquiridos durante la formación presencial y el trabajo autónomo en la materia Literatura Infantil en Lengua Inglesa y su Didáctica. Entre las opciones los estudiantes podían escoger estaban: (a) he aprendido sobre las principales contribuciones literarias de literatura infantil en lengua inglesa, (b) he conocido algunas contribuciones literarias de literatura infantil y su posible uso y aplicación en el aula, (c) he diseñado actividades en base a cuentos o poemas o (d) he mejorado las destrezas orales.

En este sentido, los resultados obtenidos indican que el $60 \%$ de los alumnos encuestados escogen la opción (b) que hace referencia al conocimiento y familiarización con las principales obras infantiles en lengua inglesa y a lo que se le añade la aplicación didáctica de estas contribuciones. Este aspecto coincide con unos de los principales objetivos de la materia descritos en el apartado 3 de este trabajo y que se refería al componente didáctico de la asignatura como vehículo para aumentar la motivación de los estudiantes.

Es significativo también que un $30 \%$ de los estudiantes señalen que han aprendido a diseñar actividades a través de cuentos y poemas, resultado que podría evidenciar la valoración positiva que los estudiantes hacen de estas dos actividades realizadas. Son solo un $10 \%$ de los encuestados los que señalan haberse familiarizado únicamente con los principales textos literarios infantiles escritos en lengua inglesa, lo que indica que la asignatura y su enfoque van más allá del mero componente teórico. 


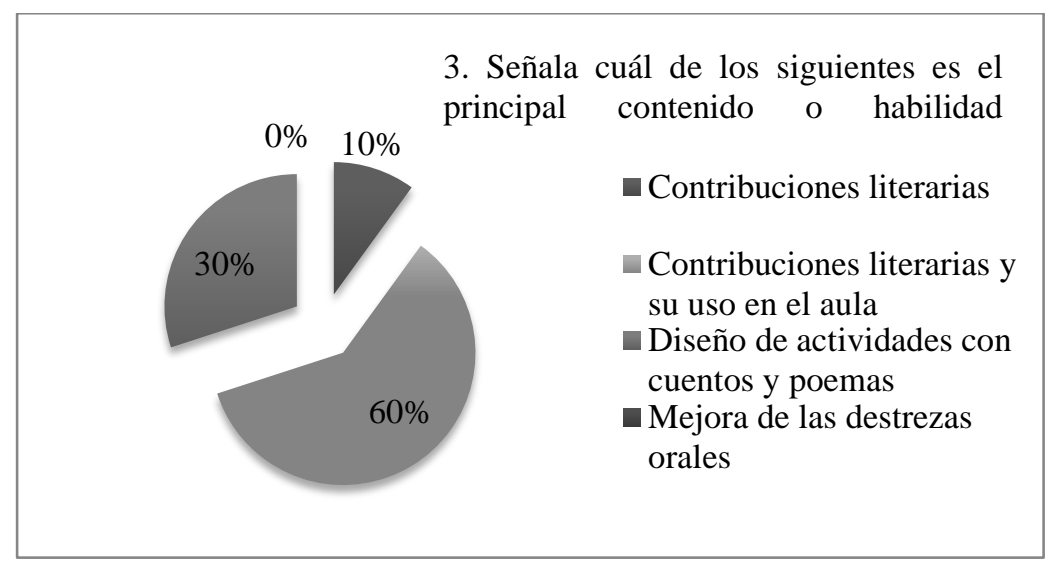

Figura 3. Pregunta 3 del cuestionario: señala cuál de los siguientes es el principal contenido o habilidad desarrollado en la materia

Preguntados si consideran imprescindible la materia para ejercer su profesión como maestros en el futuro, un $90 \%$ dice considerarla imprescindible, mientras un $10 \%$ señala que no la considera necesaria en el ejercicio de su futura profesión. El objetivo fundamental de esta pregunta tiene que ver con la adquisición y desarrollo de competencias profesionales durante su formación como maestros, aspecto muy demandado por los estudiantes desde que inician sus estudios en Educación Infantil y Primaria. De este modo, observamos que la materia contribuye de manera positiva o muy positiva para su cualificación práctica como docentes a través de las diferentes actividades con base literaria y carácter práctico presentes en la guía docente. La figura 4 muestra estos porcentajes.

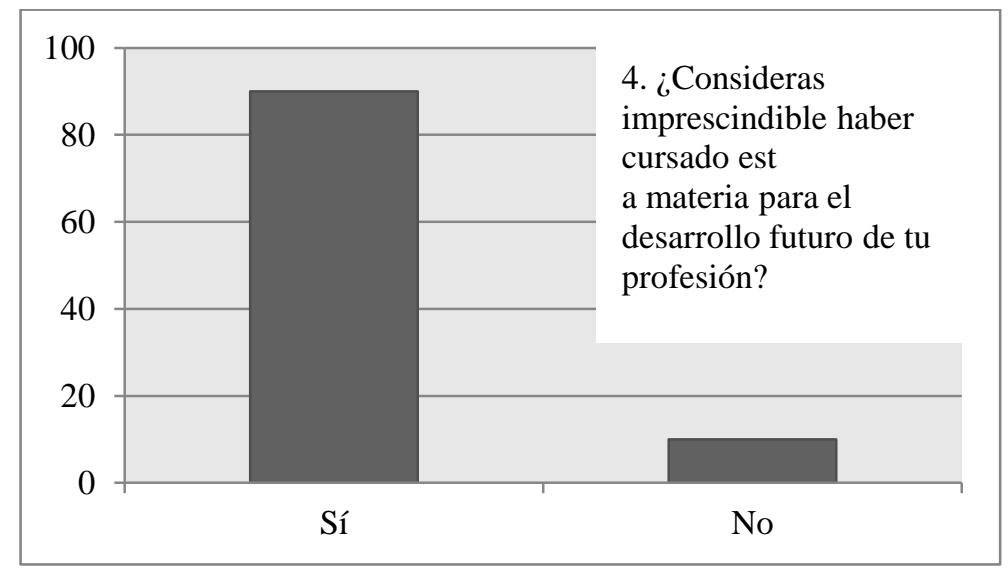

Figura 4. Respuesta 4 del cuestionario: ¿Consideras imprescindible haber cursado esta materia para el desarrollo futuro de tu profesión?

Por último, pretendíamos conocer si los estudiantes han hecho uso de las lecturas obligatorias realizadas en Literatura Infantil en Lengua Inglesa y su Didáctica en otras materias cursadas por estos durante el mismo período. Son un $70 \%$ los que sí aseguran haber hecho uso de estas lecturas, mientras que un 30\% señala no haber utilizado tales contribuciones como base para la preparación de actividades en otras materias cursadas como muestra la figura 5.

ISL, vol. 4, 2015, págs. 93-106 ISNN: 2340-8685
Guadamillas Gómez, M. V. (2015): La formación de los futuros maestros a través de la literatura: los textos literarios desde una perspectiva didáctico-práctica, Investigaciones Sobre Lectura, 4, 93-106. 


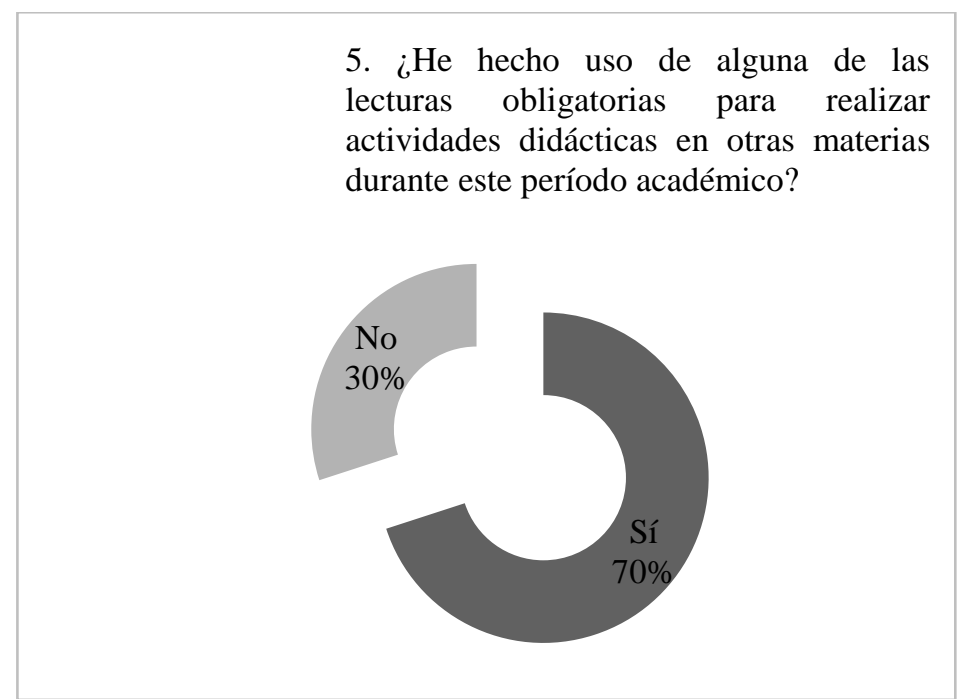

Figura 5. Respuesta 5 del cuestionario: ¿He hecho uso de alguna de las lecturas obligatorias para realizar actividades didácticas en otras materias durante el mismo curso académico?

Esta última pregunta nos permitía comprobar no solo la posible formación práctica adquirida en la asignatura y que podría ser relevante para el ejercicio de la profesión docente, tratada en la pregunta 4 del cuestionario, sino la transferencia de la materia a otras asignaturas cursadas por los alumnos y que tienen que ver, en concreto, con metodología y didáctica de la lengua inglesa. Vemos que los textos leídos no solo podían aplicarse en el aula, sino también llevarse a otras áreas formativas.

Por último, conviene destacar algunas de las respuestas de los estudiantes, cuando estos fueron preguntados sobre los aspectos positivos y negativos observados en el desarrollo de la asignatura. De los 20 alumnos, 18 de ellos consideró negativo: "haber tenido que realizar muchos trabajos, actividades, lecturas...etc.". Los dos alumnos restantes señalaron no haber encontrado ningún aspecto negativo. Respecto a las actividades o aspectos valorados más positivamente, destaca el uso de las nursery rhymes para preparar intervenciones didácticas que traten de enseñar pronunciación, ritmo y entonación en los primeros niveles. Seis de los alumnos encuestados subrayan que lo que más les satisfizo es haber podido conocer algunos autores británicos y leer libros ilustrados sencillos que podrán usar con los estudiantes en el aula.

Los porcentajes obtenidos en las cinco preguntas globales permiten afirmar la satisfacción de los estudiantes con el enfoque, la metodología y los procedimientos llevados acabado durante su formación. Del mismo modo, dichas respuestas confirman también las conexiones entre los contenidos tratados y las actividades realizadas y el ejercicio de su profesión de docentes en el futuro. Con respecto a la respuesta abierta, los encuestados nos confirman que han encontrado numerosos aspectos positivos en la materia y solo se encuentran descontentos con las numerosas intervenciones que debían desarrollar en el aula. 


\subsection{Discusión de resultados}

Una vez presentados los resultados de la investigación, este apartado reflexionará sobre la posible respuesta que estos aportan a los objetivos de nuestro estudio, así como sobre otras implicaciones más amplias que tienen que ver con la formación de los maestros en literatura y el incremento de la motivación de estos a través de los textos literarios. Atenderemos también a los resultados obtenidos en la última pregunta, de carácter abierto, y en la que los encuestados debían indicar un aspecto positivo y otro negativo de la formación recibida.

El primero de nuestros objetivos era conocer la importancia que los estudiantes daban a Literatura Infantil en Lengua Inglesa y su Didáctica dentro del plan de estudios de Maestro en Educación Infantil. Podemos decir que los resultados obtenidos fueron muy positivos, y que estos se alejan de percepciones generalizadas tales como la falta de interés de los alumnos por la formación literaria o el descontento hacía las asignaturas que exigen lecturas obligatorias. Destacamos que ningún alumno consideró la materia como poco importante, considerando que se trató de cuestionarios anónimos. El segundo objetivo de nuestro estudio era mucho más general y pretendía abordar la cuestión de si los estudiantes incrementaban a través de la materia su interés por la literatura infantil escrita en lengua inglesa. En este caso, todos los estudiantes respondieron positivamente de manera unánime, si bien, cabe destacar, que no nos paramos en este cuestionario a analizar su género o subgénero favorito. Es decir, algunos estudiantes pudieron disfrutar de la lectura de libros ilustrados y no hacerlo de textos narrativos como los escritos por Roald Dahl, sin embargo, todos aumentaron su interés por estos textos gracias a alguna de las lecturas realizadas.

En tercer lugar, nos proponíamos conocer cuáles de las propuestas eran las competencias o habilidades que los estudiantes habían adquirido a través de esta asignatura y teniendo en cuenta su enfoque. En este caso, las respuestas fueron menos uniformes, pero sí podemos afirmar que la mayoría destacó, no solo haber leído y disfrutado de algunos textos infantiles escritos en lengua inglesa, sino también haber aprendido cómo podían hacer uso de ellos en el aula. Es cierto que los estudiantes solo podían marcar una de las cuatro opciones planteadas y, podría considerarse, que en algunos casos hubiesen desarrollado más de una habilidad o competencia.

Para nuestro cuatro objetivo: (4) conocer las conexiones entre lo tratado en esta materia y el desarrollo de su profesión como maestros, los resultados obtenidos muestran que, en general, el alumnado considera muy relevante haber cursado la asignatura para enfrentarse a su futura profesión docente que comenzará con su experiencia práctica como alumnos en los centros educativos. Esto es debido, en parte, al enfoque didáctico seguido en el desarrollo de las diferentes actividades de aula tales como la presentación y desarrollo de intervenciones didácticas a partir de poemas cortos o libros ilustrados o la dramatización de cuentos infantiles breves en lengua inglesa.

ISL, vol. 4, 2015, págs. 93-106 ISNN: 2340-8685
Guadamillas Gómez, M. V. (2015): La formación de los futuros maestros a través de la literatura: los textos literarios desde una perspectiva didáctico-práctica, Investigaciones Sobre Lectura, 4, 93-106. 
Dichas intervenciones, para las que los alumnos elaboraron material propio, pueden llevar la literatura infantil al aula en sus intervenciones reales.

Por último, el cuestionario diseñado trataba de indagar sobre el uso que los alumnos podían darle al material literario tratado en otras asignaturas similares cursadas durante el mismo curso académico, para lo cual obtuvimos respuestas bastante positivas que indican la interdisciplinariedad de la literatura, en general, y de esta materia, en particular. En este sentido, debemos señalar que diferentes áreas del curriculum del segundo ciclo de Educación Infantil pueden ser tratadas a través de textos literarios a los que los estudiantes tuvieron acceso gracias a la asignatura objeto de estudio.

Finalmente, entre los aspectos positivos señalados por los encuestados, destaca, la posibilidad que los estudiantes dicen haber tenido a través de la materia de acercarse a los libros ilustrados. Ejemplos como The Gruffalo o The Very Hungry Caterpillar, eran desconocidos para los alumnos hasta ese momento. Asimismo, destacan también otros comentarios que subrayan la posibilidad de haber aprendido como hacer uso de nursery rhymes populares para enseñar la pronunciación en L2 a los estudiantes de Educación Infantil. Entre los aspectos negativos de la materia más señalados por los estudiantes se encuentra la carga de trabajo que la asignatura conlleva: trabajos, presentaciones, etc. A este respecto, hemos de tener en cuenta que la materia tenía carácter presencial y los estudiantes debían presentar sus intervenciones, dramatizaciones o sesiones quincenalmente.

En general, los resultados obtenidos indican una alta satisfacción y un incremento de la motivación a través de la asignatura Literatura Infantil en Lengua Inglesa y su Didáctica en los estudiantes del Grado de Maestro en Educación Infantil. Todo ello nos permite considerar que el enfoque es adecuado y el componente didáctico necesario en la formación de maestros futuros. Con todo ello, podemos considerar que el estudio nos permite no solo mostrar el enfoque seguido en la materia objeto de estudio y que puede ser útil para otros contextos de educación superior similares al descrito, sino que también da muestra de lo llamativo y provechoso que la práctica literaria educativa puede resultar a los estudiantes de Educación. Por último, debemos destacar que aunque el número de participantes en el estudio es reducido, no por ello es menos significativo, ya que aporta detalle y concreción al presente trabajo. En este sentido, trabajos futuros podrían dirigir la atención a aumentar el número de parcipantes y el contexto al que estos pertenecen para poder aportar unos resultados más generales a esta investigación.

\section{Conclusiones}

Este artículo ha estudiado el enfoque de la materia Literatura Infantil en Lengua Inglesa y su Didáctica dentro del Grado de Maestro de Educación Infantil según el plan de estudios vigente para el curso 2014-2015. En concreto, se ha referido a la 
combinación metodológica teórico-práctica que nos ha permitido que los futuros maestros incrementen su interés y motivación por la lectura, en este caso, en lengua inglesa. Se ha fijado también en la importancia de establecer conexiones entre la práctica didáctica diaria que será llevada a cabo por los futuros profesionales y el uso de materiales literarios tales como los libros ilustrados, los poemas o rimas breves o el teatro.

En primer lugar, se ha estudiado desde una perspectiva teórica la importancia de la formación literaria para los maestros. Se han considerado aportaciones relevantes como las de Maley (2001), referentes a las numerosas ventajas que la literatura puede incluir en el aula o las de Cameron (2001), quien se refiere a la literatura como vehículo para la adquisición de la lengua materna y el aprendizaje de la L2. Hemos tenido también en cuenta en este apartado el curriculum vigente en Educación Infantil en lo que se refiere al uso de formas literarias en el aula, concluyendo a través de los diferentes documentos consultados, la importancia del uso de los textos literarios en etapas educativas iniciales.

En segundo lugar, este artículo ha descrito la asignatura en la que se ha llevado a cabo este estudio: Literatura Infantil en Lengua Inglesa y su Didáctica. Se ha referido a los objetivos de la materia, el desarrollo de contenidos, las actividades propuestas al alumnado y el proceso de evaluación que se ha seguido en esta. Asimismo, hemos hecho referencia a las competencias y habilidades que a través de la materia pretendíamos mejorar en nuestros alumnos. A continuación, se ha descrito el diseño de la investigación, siendo esta de carácter cualitativo y descriptivo, el número de participantes y otros aspectos que tienen que ver con el diseño de los cuestionarios.

En el apartado de análisis de resultados hemos pretendido conocer el grado de satisfacción de los participantes en torno a las cinco preguntas cerradas que les han sido realizadas, los resultados han sido discutidos, constatando, que estos son bastante o muy positivos en líneas generales y que además de cumplir con los objetivos de nuestra investigación, están también en consonancia con los objetivos que nos proponíamos al inicio de nuestro estudio. Asimismo, y a través de la respuesta abierta de los estudiantes, hemos obtenido resultados interesantes sobre, lo que a la vista de los participantes, son aspectos positivos y negativos del enfoque llevado a cabo. Hemos discutido también la importancia de la respuesta al cuestionario por parte de los estudiantes, apuntando que estos representan una herramienta de reflexión y evaluación tanto para sí mismos como para su formador.

No se trata solo de un estudio aislado, sino que se ha pretendido presentar también un enfoque clínico en la formación de los futuros profesionales que, a través de la intervención individual o en pequeños grupos, les permita motivarse con la lectura, de manera general y, en concreto, con los textos infantiles escritos en lengua inglesa. Esto 
es de gran importancia, si tenemos en cuenta que el desconocimiento de determinados materiales literarios es la causa directa de que estos caigan en desuso en aulas que cada vez demandan más la práctica literaria desde los inicios.

Esta contribución supone, por lo tanto, no solo un modelo para los docentes interesados en trabajar la literatura en lengua inglesa en otras Facultades de Educación, sino también, un análisis de la práctica docente llevada a cabo durante el primer cuatrimestre del curso académico 2014-2015 y que, en líneas generales, ha contado con un gran respaldo por parte del alumnado, ya que le ha permitido acercarse al uso de la literatura escrita en lengua inglesa en el aula de Educación Infantil e incrementar su motivación a través de un aprendizaje activo, continuo y colaborativo.

\section{Bibliografía}

Andreucci Anunciata, P. (2012). El enfoque clínico en la formación continua de profesores: la teorización de "ojo pedagógico" como destreza compleja. Revista de currículum y formación de profesorado, 16, 258-275.

Caballud, M. y Carramiñana Ibañez, C. (2015). La formación del profesorado, las familias y los planes de estudio de lectura y escritura. Textos didácticos de la lengua y la literatura, 68, 28-36.

Cameron. L. (2001). Teaching Languages to Young Learners. Cambridge: Cambridge University Press.

Carle, E. (1986). The Very Hungry Caterpillar. Nueva York: Collins Publishers.

Donaldson, J. y Scheffer, A. (1999). The Gruffalo. Londres: MacMillan Children's Books.

España. Real Decreto 67/2007, de 29 de diciembre, por el que se establecen las enseñanzas mínimas del segundo ciclo de Educación Infantil. Boletín Oficial del Estado, 4 de enero de 2007, núm. 4, (pp. 474-482).

Hernández Castilla, R. y Opazo Carvajal, H. (2010). Apuntes de Análisis Cualitativo en Educación. Recuperado el 30 de mayo, 2015 de http://www.uam.es/personal_pdi/stmaria/jmurillo/Met Inves Avan/Materiales/ Apuntes_Cualitativo.pdf 
Khatib, M., Derakhshan, A y Rezaei, S. (2011). Why and Why Not Literature: A Taskbased approach to teaching literature. International Journal of English Linguistics, 1 (1), 212-220.

Maley, A. (2001). Literature in the language classroom. En R. Carter y D. Nunan (Coords.), The Cambridge guide to teaching English to speakers of other languages (pp. 180-185). Cambridge: Cambridge University Press.

Murphy, J. (2007) On The Way Home. Londres: Macmillan Children's Books.

Selfa i Sastre, M., Fraga de Azevedo, F. J. y Berengué Carbonell, I. (2015). Leer sobre la muerte en una Biblioteca de Aula: una experiencia práctica de lectura en el aula de Educación Infantil. Investigaciones Sobre Lectura, 3, 83-95.

Van, T. M. (2009). The relevance of literary analysis to teaching literature in the EFL classroom. English Teaching Forum, 3, 2-9.

Villacañas de Castro, L. (2013). Una didáctica interdisciplinar de la literatura infantil en inglés. Didáctica de la lengua y la literatura, 25, 353-373.

ISL, vol. 4, 2015, págs. 93-106 ISNN: 2340-8685
Guadamillas Gómez, M. V. (2015): La formación de los futuros maestros a través de la literatura: los textos literarios desde una perspectiva didáctico-práctica, Investigaciones Sobre Lectura, 4, 93-106. 